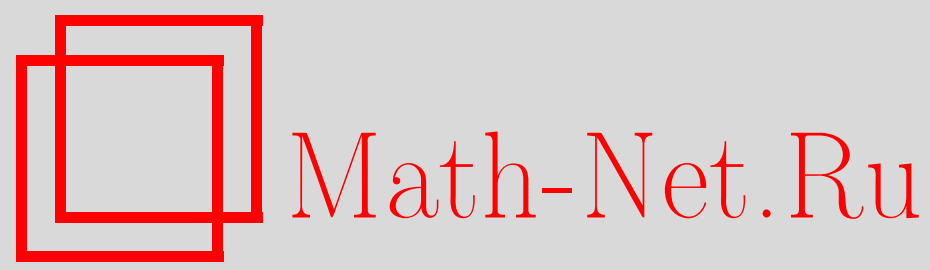

О. В. Бородин, Д. Р. Вудал, Вес граней в плоских картах, Матем. заметки, 1998, том 64, выпуск 5, 648-657

DOI: https://doi.org/10.4213/mzm1441

Использование Общероссийского математического портала Math-Net.Ru подразумевает, что вы прочитали и согласны с пользовательским соглашением http://www.mathnet.ru/rus/agreement

Параметры загрузки:

IP: 44.207 .124 .84

26 апреля 2023 г., 17:01:28

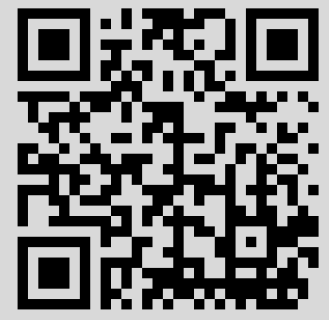


УДК 519.17

\title{
ВЕС ГРАНЕЙ В ПЛОСКИХ КАРТАХ
}

\author{
О.В. Бородин, Д. Р. Вудал
}

\begin{abstract}
Найдены точные верхние оценки для минимального веса младшей грани в плоских нормальных картах и 3-мерных выпуклых многогранниках с заданной максимальной степенью вершины.

Библиография: 12 названий.
\end{abstract}

1. Введение. Работа посвящена исследованию комбинаторного строения плоских графов. Как доказано Штейницом [1], 3-связные плоские графы взаимно однозначно соответствуют вьпукльп 3-мерным многогранникам, именуемым далее 3-многогранниками.

В развитии этой области большую роль сыграла теория эйлеровых вкладов, предложенная Лебегом [2] для получения структурной информации о плоском графе. Одним из стимулов для этой и последуюших работ являлась проблема четырех красок. При всей своей универсальности и идейной простоте подход Лебега не обладает достаточной разрешающей способностью, чтобы ответить на многие естественные вопросы о строении и раскрасках плоских графов.

В последние десять лет в данной области наблюдается значительньй прогресс. Так, Бородиным (см. [3]) получен ряд результатов о строении окрестностей ребер и граней в различных классах плоских графов, имеющих приложения к задачам цикловой, совместной и предписанной раскрасок. На основе единого метода в [3] решены некоторые задачи, поставленные в 60-70 гг. Рингелем, Коцигом, Грюнбаумом, Юцовичем, Пламмером, Кронком и др.

Подробное описание окрестности ребра можно найти в [4]; в настоящей работе мы рассматриваем окрестности другого базисного элемента плоских граней - граней. (В силу вершинно-граневой двойственности результаты о строении граней легко переводятся на язьк вершин и обратно.) Точнее, завершая цикл исследований, восходящий к Лебегу [2], мы получаем точную верхнюю оценку для суммы степеней граничных вершин грани в 3-многогранниках через максимальную степень вершины.

Перейдем к определениям. Те из них, которые касаются правильных и полуправильных многогранников (платоновых и архимедовых тел), можно найти в [5].

$C$ тепенью $d(v)$ вершины $v$ (степенью $r(f)$ грани $f$ ) в графе $G$ назьвается число инцидентных ей ребер; $k$-вершиной ( $k$-гранью) называется вершина (грань) степени $k$, $\mathrm{a} \geqslant k$-вершина имеет степень не меньше $k$, и т.д.

Работа первого автора выполнена при финансовой поддержке Британского Совета по физикотехническим наукам, грант GR/K00561, и Российского фонда фундаментальных исследований, гранты № 96-01-01614 и № 97-01-01075. 
Как известно, каждая нормальная плоская карта, т.е. такая, в которой каждая вершина и каждая грань имеет степень не меньше 3, содержит младиую грань, т.е. грань степени не больше 5 . В дальнейшем $M$ всегда обозначает плоскую нормальную карту с максимальной степенью $\Delta=\Delta(M)$. Мы ограничиваемся рассмотрением нормальных карт, поскольку иначе может вообще не быть младших граней, как показьвает пример цикла $C_{n}$. Вес грани в $M$ есть сумма степеней окружающих вершин, а $w(M)$ (или просто $w)$ обозначает минимальный вес младией грани в $M$.

Лебег [2] получил теорему о строении младших граней в $M$, из которой, в частности, следует, что $w \leqslant \max \{51, \Delta+9\}$. При $\Delta \geqslant 42$ эта оценка точна, как следует из $\Delta$-угольной антипризмы (см. ниже). Кроме того, результат Лебега влечет точные верхние оценки для $w$ при $\Delta \leqslant 10$, включенные нами в следующую ниже теорему 1 .

Для триангулированных 3-многогранников без 4-вершин Коциг [6] доказал оценку $w \leqslant 39$, а Бородин [7] установил, что $w \leqslant 29$, и эта оценка является неулучшаемой ввиду дважды усеченного додекаэдра. Нетриангулированный 3-многогранник может иметь сколь угодно большой вес $w$, как показьвает $n$-пирамида. С другой стороны, при наличии 4-вершин даже триангулированньй многогранник может иметь неограниченный вес $w$, как показьвает двойная $n$-пирамида. Бородин [8] далее установил, что если в триангулированном 3-многограннике никакая 4-вершина не смежна с 3- или 4-вершинами, то по-прежнему $w \leqslant 29$, а если запретить лишь 4,4 -ребра, то имеет место точная оценка $w \leqslant 37$. Поскольку наличие $\leqslant 4, \leqslant 4$-ребра сразу дает $w \leqslant \Delta+8$, отсюда следует, что каждьй триангулированньй 3 -многогранник имеет вес $w \leqslant \max \{29, \Delta+8\}$. Заметим, что в двойной $\Delta$-пирамиде каждая грань имеет вес $\Delta+8$, так что обе компоненты в последней оценке неулучшаемы.

Для произвольных $M$ оценка $w \leqslant \Delta+9$ является неулучшаемой всюду, где она верна: возьмем двойное $(2 \Delta)$-колесо (пирамиду) и удалим верхние спищы с четными номерами и нижние с нечетньми с получением $\Delta$-угольной антипризмы, в которой каждая грань имеет тип $(3,3,3, \Delta)$. Бородин доказал [3], [9], что если в $M$ нет граней типа $(3,3,3, \leqslant \Delta)$, то $w \leqslant \max \{32, \Delta+8\}$. Хорнак и Ендроль [10] доказали с помощью компьютерных вычислений, что если $M$ не содержит $(3,3,3, \leqslant \Delta)$-, $(\leqslant 4, \leqslant 4, \leqslant \Delta)$ - и $(3,5, \leqslant 39)$-граней, то $w \leqslant 32$. В настоящей статье мы доказьваем (следствие 3$)$, что $w \leqslant \max \{29, \Delta+8\}$ либо в $M$ есть $(3,3,3, \leqslant \Delta)$-грань.

Бородин и Вудал [11] доказали, что $M$ имеет вес $w \leqslant 25$ либо содержит грань одного из следуюших типов: $(3,3, \leqslant 4, \leqslant \Delta),(\leqslant 4, \leqslant 4, \leqslant \Delta),(3,5, \leqslant \Delta),(3,6, \leqslant 20)$, $(4,5, \leqslant 19)$ или $(3,3,5, \leqslant 19)$.

Хорнак и Ендроль [10] изучали минимум $w^{*}$ веса граней по всем граням в $M$, а не только по младшим, как делалось всеми, начиная с Лебега [2]. Очевидно, $w^{*} \leqslant w$. Они показали [10], что любая карта $M$ имеет $w^{*} \leqslant \max \{32, \Delta+9\}$, а любая карта $M$ без $(3,3,3, \leqslant \Delta)-$ и $(\leqslant 4, \leqslant 4, \leqslant \Delta)$-граней имеет $w^{*} \leqslant 32$.

При $3 \leqslant k \leqslant 10$ и $k \geqslant 42$ следующая теорема 1 вытекает из результата Лебега [2]; при $11 \leqslant k \leqslant 12$ из работ Бородина [12] и Хорнака, Ендроля [10], а при $19 \leqslant k \leqslant 20$ и $24 \leqslant k \leqslant 41$ из работ Бородина [3], [9] и Хорнака, Ендроля [10].

Цель данной статьи состоит в завершении этих исследований получением точной верхней оценки для минимального веса младшей грани в нормальных картах через максимальную степень вершины.

ТЕОРема 1. Если $k \geqslant 3$, а $M$ - нормальная плоская карта максимальной степени $\Delta(G) \leqslant k$, то $M$ содерэсит младшую грань $f$ веса не более $W(k)$, где $W(k)$ неулучшаема и задается табл. 1.

Метод, использованный Бородиным [3], [9] и Хорнаком, Ендролем [10], непригоден 
ТАБЛИЦА 1

\begin{tabular}{|c|c|c|l|}
\hline$k$ & $W(k)$ & грани & \multicolumn{1}{|c|}{ двойственньй многогранник } \\
\hline 3 & 15 & $12\left(3^{5}\right)$ & к икосаэдру (т.е. додекаэдр) \\
4 & 16 & $24\left(3^{4} .4\right)$ & к плосконосому кубу \\
5 & 17 & $60\left(3^{4} .5\right)$ & к плосконосому додекаэдру \\
6 & 17 & $60\left(5.6^{2}\right)$ & к усеченному икосаэдру \\
7 & 18 & $64\left(4.7^{2}\right), 8\left(7^{3}\right)$ & к конструкции 1 (см. текст) \\
8 & 19 & $24\left(3.8^{2}\right)$ & к усеченному кубу \\
9 & 21 & $48\left(3.9^{2}\right), 6\left(3^{2} 9^{2}\right)$ & к конструкции 2 \\
10 & 23 & $60\left(3.10^{2}\right)$ & к усеченному додекаэдру \\
11 & 24 & $120(3.10 .11), 40\left(3.11^{2}\right), 10\left(3^{2} 11^{2}\right)$ & к конструкции 3 \\
$12-16$ & 25 & $120(3.10 .12), 60\left(3.12^{2}\right)$ & к дважды усеченному икосаэдру \\
$\geqslant 17$ & $k+9$ & $2 k\left(3^{3} k\right)$ & к конструкции 4 \\
\hline
\end{tabular}

для разрешения оставшихся в теореме 1 случаев. Фактически мы даем единое доказательство для всех $k \geqslant 11$, основанное на нашем подходе в [11], и используем результат Лебега [2] при $k \leqslant 10$.

В табл. 1 приведены многогранники, подтверждающие неулучшаемость оценок теоремы 1. Здесь $24\left(3^{4} .4\right)$, например, означает наличие в многограннике 24 граней, каждая из которых граничит с одной 4-вершиной и четырьмя 3 -вершинами, так что каждая грань имеет вес 16.

Табл. 1 дополняется следующими конструкциями.

Конструкция 1. Усекаем все 4-вершины в двойственном многограннике к ромбокубооктаэдру и берем двойственный многогранник.

Конструкция 2. Стягиваем шесть ребер в усеченном додекаэдре, которые совместно инцидентны всем двенадцати 10-граням.

Конструкция 3. Стягиваем десять ребер в дважды усеченном икосаэдре, которые совместно инцидентны всем двадцати 12-граням, и берем двойственньй многогранник.

Конструкция 4. Приводим двойственньй многогранник к $k$-угольной антипризме.

Вместо теоремы 1 нам будет удобнее доказать следующую ниже более сильную теорему 2 , в которой параметры 25 и 24 являются неулучшаемыми. Будем говорить, что грань имеет mun $(a, \leqslant b, \geqslant c)$ или является $(a, \leqslant b, \geqslant c)$-гранью, если инцидентные ей вершины имеют степени (вне какого-либо определенного порядка) $d_{1}=a, d_{2} \leqslant b$ и $d_{3} \geqslant c$; остальные типы определяются аналогично.

ТЕОрема 2. Пусть $M-$ нормальная плоская карта с $\Delta(M)=\Delta$, не содержа$u_{1}$ ая граней типа $(\leqslant 4, \leqslant 4, \leqslant \Delta),(3,5, \leqslant \Delta),(3,3,3, \leqslant \Delta) u(3,6, \leqslant 20)$. Тогда $M$ содерэит младшую грань, отличную от $(3,11,11)$-грани, вес которой не более 25 при $\Delta \geqslant 12$ и не более 24 при $\Delta=11$.

Ни один из четырех запрещенных типов граней не может быть опущен в формулировке теоремы 2 , благодаря соответственно $\Delta$-угольной антипризме, построенному Хорнаком и Ендролем [10] 3-многограннику с $w=26$ без граней типов $(\leqslant 4, \leqslant 4, \leqslant \Delta)$, $(3,3,3, \leqslant \Delta)$ и $(3,6, \leqslant 20), \Delta$-угольной антипризме и многограннику, двойственному к дважды усеченному додекаэдру.

Веса граней четырех запрещенных в теореме 2 типов не превосходят $\Delta+8, \Delta+8$, $\Delta+9$ и $\min \{29, \Delta+9\}$ соответственно. Поэтому (с учетом результатов Лебега [2] при 
$k \leqslant 10$ и конструкций из табл. 1) теорема 1 легко следует из теоремы 2.

Теорема 2 влечет следующий результат, в котором все параметры, как уже отмечалось, являются неулучшаемьми.

СледСТВИЕ 3. Каждая нормальная карта без $(3,3,3, \leqslant \Delta)$-граней имеет вес $w \leqslant \max \{29, \Delta+8\}$.

2. Доказательство теоремы 2. Предположим, что $M=(V, E, F)$ - контрпример к теореме 2 , имеющий минимально возможное число вершин степени больше 3 и (c выполнением этого ограничения) максимизирующий число ребер, соединяющих такие вершины, плюс число 3-граней, инцидентных по меньшей мере двум таким вершинам. Мы будем часто пользоваться следующей леммой.

ЛЕмма 1. а) Пусть иvш - три последовательные вериины вдоль границь нетреугольной грани $f$ в $M$. Тогда

$$
d(w) \leqslant \begin{cases}19 & n p u d(u)=5 \\ 9 & n p u d(u) \geqslant 10\end{cases}
$$

а если $6 \leqslant d(u) \leqslant 9, \operatorname{mo} d(u)+d(w) \leqslant 20$.

б) $B M$ нет 3 -граней $f$ muпа $(5, \geqslant 20, \geqslant 20),(6, \geqslant 15, \geqslant 15),(7, \geqslant 14, \geqslant 14)$, $(8, \geqslant 13, \geqslant 13),(9, \geqslant 12, \geqslant 12) u(\geqslant 10, \geqslant 10, \geqslant 10)$.

ДокАЗАтЕльство. Если $f$ нарушает утверждение а), то добавим ребро $u w$ внутрь $f$ с получением карты $M^{*}$, в которой либо $d(u)=6$ и $d(w) \geqslant 21$, либо $d(u) \geqslant 11$ и $d(w) \geqslant 11$, либо $d(u) \geqslant 7, d(w) \geqslant 7$ и $d(u)+d(w) \geqslant 23$. При этом не возникает граней ни одного из четырех запрешенных типов. Веса старых граней не уменьшились, в то время как всякая новая грань имеет либо тип $(3,11,11)$, либо вес не менее $23+3=26$. Отсюда $M^{*}$ является контрпримером к теореме 2 , и в ней больше чем в $M$ ребер, соединяющих вершины степени больше 3 , что противоречит выбору $M$.

Если $f$ нарушает утверждение б), то вставим внутрь $f$-вершину, смежную с $u, v$ и $w$. Новые треугольники имеют типы $(3, \geqslant 6, \geqslant 21),(3, \geqslant 7, \geqslant 16),(3, \geqslant 8, \geqslant 15)$, $(3, \geqslant 9, \geqslant 14),(3, \geqslant 10, \geqslant 13)$ или $(3, \geqslant 11, \geqslant 11)$. Поэтому мы снова имеем контрпример, противоречащий выбору $M$.

Теперь предположим, что мыприписьваем заряд $M(x)$ каждому элементу $x \in V \cup F$, где

$$
M(x):= \begin{cases}2 d(x)-6, & \text { если } x \in V, \\ r(x)-6, & \text { если } x \in F .\end{cases}
$$

Формула Эйлера $|V|-|E|+|F|=2$ может быть переписана в виде $(4|E|-6|V|)+$ $(2|E|-6|F|)=-12$, откуда следует, что

$$
\sum_{x \in V \cup F} M(x)=\sum_{v \in V}(2 d(v)-6)+\sum_{f \in F}(r(f)-6)=-12 .
$$

Теперь мы перераспределим заряды, не меняя их суммы, таким образом, что их сумма станет доказуемо неотрицательной, и это противоречие завершит доказательство теоремы. Правила перераспределения зарядов следуюшие.

Правило R1. Каждая 4-вершина дает $\frac{1}{2}$ каждой инщидентной грани.

Правило R2. Каждая 5-вершина $v$ дает $\frac{1}{2}$ каждой инцидентной грани за тем исключением, что дает

a) 0 нетреугольной грани ...uvw .., если $d(u)=d(w)=4$; 
б) $\frac{1}{4}$ нетреугольной грани ...uvw .., если $d(u)=5, d(w)=4$;

в) $\frac{3}{4}$ грани типа $(5,5, \leqslant \Delta)$;

г) 1 грани типа $(4,5, \leqslant \Delta)$;

д) $0(\geqslant 16,5, \geqslant 16)$-грани.

Правило R3. Каждая $\geqslant 6$-вершина $v$ дает 1 каждой инцидентной нетреугольной грани за тем исключением, что дает

а) $\frac{3}{2}$ грани $u v w x$, если $d(v) \geqslant 11$, и грани $u w v x$, если $d(v) \geqslant 12$,где в обоих случаях $4 \leqslant d(u) \leqslant 9$ и $d(w)=d(x)=3$;

б) $\frac{1}{2} \geqslant 4$-грани ...uvw..., если $d(u) \leqslant 5, d(v)=7$ и $d(w) \leqslant 5$;

в) $\frac{3}{4} \geqslant 4$-грани ...uvw..., если $d(u) \leqslant 5, d(v)=7$ и $6 \leqslant d(w) \leqslant 13$;

г) $\frac{7}{5}$, если $d(v)=10$.

Правило R4. Каждая $\geqslant 6$-вершина $v$ дает 1 каждой инщидентной 3-грани $u v w$, если $d(u) \geqslant 6$ и $d(w) \geqslant 6$. Если $d(u) \leqslant 5$ или $d(w) \leqslant 5$, то для $d(v)=6, d(v)=7, d(v)=8$, $d(v)=9, d(v)=10$ и $d(v) \geqslant 11$ вершина $v$ дает

$$
\begin{cases}1,1,1, \frac{4}{3}, \frac{7}{5}, \frac{3}{2}, & \text { если } \Delta=11, \\ 1, \frac{5}{4}, \frac{5}{4}, \frac{4}{3}, \frac{7}{5}, \frac{3}{2}, & \text { если } \Delta \geqslant 12,\end{cases}
$$

соответственно за следуюшим исключением: если $d(u)=3$ и вторая грань $f^{*}=w^{*} u v \ldots$, инцидентная ребру $u v$, есть треугольник и $d\left(w^{*}\right) \geqslant 11$, то вершина $v$ дает 3-грани $u v w$ заряд

$$
\begin{cases}2, & \text { если } d(w)=6, \\ \frac{7}{4}, & \text { если } d(w)=7 \text { или } 8, \\ \frac{5}{3}, & \text { если } d(w)=9, \\ \frac{8}{5}, & \text { если } d(w)=10 .\end{cases}
$$

Правило R5. Если нетреугольная грань $f^{*}=z^{*} x y$... имеет общее ребро $x y$ с треугольником $f$, где $d(x)=3, d\left(z^{*}\right) \leqslant 10$ и $6 \leqslant d(y) \leqslant 10$, то $f^{*}$ передает через $x y$ на $f$ заряд

$$
\begin{cases}\frac{1}{2} & \text { при } d(y)=6, \\ \frac{1}{4} & \text { при } 7 \leqslant d(y) \leqslant 8, \\ \frac{1}{6} & \text { при } d(y)=9, \\ \frac{1}{10} & \text { при } d(y)=10 .\end{cases}
$$

Заметим, что правило R5 не может возникать при $\Delta=11$, поскольку иначе $f$ имела бы запрещенньй тип $(3, \leqslant 10, \leqslant 11)$.

Обозначив получившийся на каждом ребре $x \in V \cup F$ новый заряд через $M^{*}(x)$, имеем

$$
\sum_{x \in V \cup F} M^{*}(x)=-12
$$

по (1). Остаток доказательства состоит в проверке того, что $M^{*}(x) \geqslant 0$ для каждого $x$, с получением очевидного противоречия, завершающего доказательство теоремы. Доказательство удобно разбить на случаи.

1) $x$ есть вершина, $x=v \in V$. Положим $d:=d(v)$.

1a) $d \geqslant 13$. Для краткости грани, инцидентные $v$ и получающие от $v$ ровно $\frac{3}{2}$ и меньше $\frac{3}{2}$, будут назьваться $N$-гранями и $L$-гранями соответственно (или $N$-треугольниками и т.п.). Грани же, получаюшие больше $\frac{3}{2}$, с необходимостью являются треугольниками, возникающими в исключении к правилу $\mathrm{R} 4$, и называются $U$-треугольниками. 


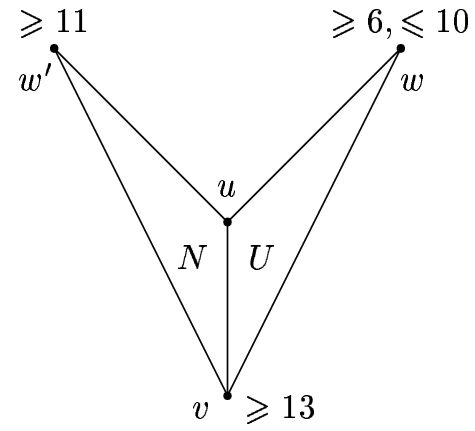

Рис. 1. Степени вершин при $N U$-парах

Также по правилу $\mathrm{R} 4$ грань, соседствующая с $U$-треугольником по ребру $u v$, является $N$-треугольником. Такая пара треугольников будет назьваться $N U$-napoй и обозначаться $\langle N \mid U\rangle$ или $w^{*}\langle N \mid U\rangle^{w}$, если нам нужно указать их "граничные" вершины. Заметим, что тогда $d\left(w^{*}\right) \geqslant 11$, a $6 \leqslant d(w) \leqslant 10$ по правилу R4 (см. рис. 1$)$.

ЛЕмма 2. Следующие последовательности типов граней не могут возникать в ииклическом порядке вокруг $v$ :

i) $\langle N \mid U\rangle^{y}\langle N \mid U\rangle$;

ii) $\langle N \mid U\rangle^{y} N^{z}\langle U \mid N\rangle$;

iii) $\langle U \mid N\rangle^{y} N^{z}\langle N \mid U\rangle$.

ДокАЗАТЕЛЬСтво. Ясно, что последовательность і) невозможна из-за степеней на рис. 1 , так как давала бы сразу $d(y) \leqslant 10$ и $d(y) \geqslant 11$. Из последовательности іi) вытекало бы $d(y) \geqslant 6$ и $d(z) \geqslant 6$; но тогда грань между ними не может быть $N$-гранью либо по правилу R3 a), либо по правилу R4. Наконец, последовательность іiі) влекла бы $d(y) \geqslant 11$ и $d(z) \geqslant 11$, что противоречит лемме 1 , поскольку несомненно $d(v) \geqslant 10$.

Пусть $l$ и $u$ - количество $L$-граней и $U$-треугольников, инцидентных $v$. Тот факт, что $U$-треугольники входят в $N U$-пары, означает, что

$$
d \geqslant 2 u+l
$$

Лемма 2 i) с оценкой $(2)$ при $l>0$ влечет, что

$$
d \geqslant 2 u+1, \text { если } u \text { нечетно, }
$$

а все части леммы 2 в совокупности при $l=0$ влекут, что

$$
d \neq 2 u+1, \text { если } u \text { четно. }
$$

Ввиду правила R4 оценка (2) доказьвает утверждение в следующих случаях:

a) при $d=14$, когда $d(w)=9$ или 10 , поскольку нет $(3, \leqslant 8,14)$-граней, так как тогда из $(2)$ и $(3)$ следует, что $u \leqslant 6$, а это влечет $M^{*}(v) \geqslant 0$ ввиду $6 \cdot \frac{5}{3}+8 \cdot \frac{3}{2}=22=$ $M(v)=2 \cdot 14-6$

б) при $16 \leqslant d \leqslant 20$, поскольку тогда $d(w) \geqslant 7$ ввиду отсутствия $(3,6, \leqslant 20)$-граней и $\frac{1}{2} d \cdot \frac{7}{4}+\frac{1}{2} d \cdot \frac{3}{2} \leqslant 2 d-6 ;$

в) при $d \geqslant 24$, поскольку тогда $\frac{1}{2} d \cdot 2+\frac{1}{2} d \cdot \frac{3}{2} \leqslant 2 d-6$;

г) при $d=23$, поскольку $11 \cdot 2+12 \cdot \frac{3}{2}=40=2 \cdot 23-6$. 
Если $d=22$, то из $(2)$ и $(3)$ следует $u \leqslant 10$, а этого достаточно, так как $10 \cdot 2+12 \cdot \frac{3}{2}=38=$ $2 \cdot 22-6$. Если $d=21$, то либо $l \geqslant 1$ и $u \leqslant 10$ по (2), либо $l=0$ и $u \leqslant 9$ по (4), чего также достаточно, поскольку $v$ дает не более 1 каждой $L$-грани и $10 \cdot 2+10 \cdot \frac{3}{2}+1=9 \cdot 2+12 \cdot \frac{3}{2}=$ $36=2 \cdot 21-6$. Аналогично, если $d=13$, то либо $l \geqslant 1$ и $u \leqslant 6$ по $(2)$, либо $l=0$ и $u \leqslant 5$ по (4), что является достаточным, поскольку $6 \cdot \frac{8}{5}+6 \cdot \frac{3}{2}+1=19.6<5 \cdot \frac{8}{5}+8 \cdot \frac{3}{2}=20=$ $2 \cdot 13-6$. Наконец, если $d=15$, то $u \leqslant 7$ по (2). Этого достаточно при $l>0$, поскольку $7 \cdot \frac{7}{4}+7 \cdot \frac{3}{2}+1=23.75<24=2 \cdot 15-6$. Также не возникает осложнений, если $l=0$ и $u \leqslant 6$, так как $6 \cdot \frac{7}{4}+9 \cdot \frac{3}{2}=24$. Но если $l=0$ и $u=7$, то по лемме 2 последовательность типов граней в циклическом порядке вокруг $v$ должна включать три последовательные $N U U N$-четверки, соединенные через $N U N$, как в $\ldots\langle N \mid U\rangle\langle U \mid N\rangle\langle N \mid U\rangle^{y} N^{w^{\prime}}\langle N \mid U\rangle^{w}\langle U \mid N\rangle \ldots$. . Здесь $d(y) \geqslant 8$ ввиду отсутствия $(3, \leqslant 7$, $15)$-граней и $d\left(w^{\prime}\right) \geqslant 11$ по правилу R4 (см. рис. 1 ), так что $N$-грань между $y$ и $w^{\prime}$ противоречит правилам R3 и R4. Этим завершается рассмотрение подслучая 1a).

1б) $d=12$ или 11. В этом случае $v$ дает каждой инцидентной грани $\frac{3}{2}$ или $\leqslant 1$. Если $d=12$, то $M(v)=2 \cdot 12-6=18$ и $v$ дает не более $12 \cdot \frac{3}{2}=18$, поэтому $M^{*}(v) \geqslant 0$. Если $d=11$, то $M(v)=2 \cdot 11-6=16$. Поскольку $10 \cdot \frac{3}{2}+1=16$, осложнение может возникать лишь когда $v$ дает $\frac{3}{2}$ каждой инцидентной грани. Но $v$ может давать $\frac{3}{2}$ инцидентной грани $f$ двумя различными способами: по правилу R3a) и по правилу R4. Ввиду отсутствия $(3,3, \leqslant 5,11)$ - и $(\leqslant 5, \leqslant 5,11)$-граней соответственно в каждом из случаев одно из ребер при $v$, граничащее с $f$, ведет в вершину степени $\leqslant 5$, а другое в вершину степени $\geqslant 6$. Очевидно, что такое невозможно, поскольку $d=11$ нечетно.

1в) $6 \leqslant d \leqslant 10$. В этом случае $v$ дает каждой инцидентной грани не больше максимума, описанного в правиле R4, или 1 в противном случае. Для любого четного $d$ этот максимум равен $2-6 / d$. Остается доказать утверждение при $d=9$ и 7 .

Если $d=9$, то $M(v)=2 \cdot 9-6=12$. Поскольку $v$ дает $\frac{4}{3}$ не больше чем восьми граням по правилу $\mathrm{R} 4$ (все они - треугольники типа $(\leqslant 5,9, \geqslant 11)), v$ дает в общей сложности не более $8 \cdot \frac{4}{3}+1 \cdot 1<12$.

Если $d=7$, то $M(v)=2 \cdot 7-6=8$, а $M^{*}(v) \geqslant 0$, если $v$ дает по $\frac{5}{4}$ не более чем четырем граням, поскольку $4 \cdot \frac{5}{4}+3 \cdot 1=8$. Поэтому предположим, что $v$ дает по $\frac{5}{4}$ пяти или более граням. Каждая из таких граней есть треугольник типа $(\leqslant 5,7, \geqslant 14)$ согласно отсутствию $(\leqslant 5,7, \leqslant 13)$-граней и правилу $\mathrm{R} 4$. Если при $v$ имеется шесть таких треугольников, то седьмая грань содержит три последовательные вершины $u v w$ со степенями $(\geqslant 14,7, \geqslant 14)$ или $(\leqslant 5,7, \leqslant 5)$. Первое невозможно по лемме 1 . Поэтому ввиду правила R3б) $v$ дает этой грани только $\frac{1}{2}$, что дает в сумме $6 \cdot \frac{5}{4}+\frac{1}{2}=8$. Те же рассуждения верны, если $v$ дает $\frac{5}{4}$ пяти граням, а две оставшиеся грани не соседствуют, поскольку тогда эти пять граней разбиваются как $4+1$ или $3+2$, и $v$ должна давать $\frac{1}{2}$ одной из оставшихся граней. Поэтому предположим, что $v$ дает $\frac{5}{4}$ пяти последовательньм граням, и пусть $v x, v y, v z$ будут (в этом порядке) тремя ребрами при $v$, не разделяющими пары этих ребер. Тогда $d(x) \leqslant 5$ и $d(z) \geqslant 14$, или наоборот. Поэтому $d(y) \leqslant 13$ по лемме 1 и имеется грань, с необходимостью нетреугольная ввиду отсутствия $!(\leqslant 5,7, \leqslant 13)$-граней, которая получает не более $\frac{3}{4}$ от $v$ по правилу R $\left.3 б\right)$ или R3в). Таким образом, $v$ раздает в сумме не более $5 \cdot \frac{5}{4}+\frac{3}{4}+1=8$.

1 г) $3 \leqslant d \leqslant 5$. Если $d=3$, то $M^{*}(v)=M(v)=0$, и если $d=4$, то $M^{*}(v)=2-4 \cdot \frac{1}{2}=0$ по правилу R1. Поэтому предположим, что $d=5$. Тогда $M(v)=2 \cdot 5-6=4$ и никакое ребро не получает от $v$ больше 1 .

Очевидно $M^{*}(v) \geqslant 0$, если только $v$ не дает по $\frac{3}{4}$ или 1 четырем или более треугольникам согласно правилам R2в) и R2г). Каждьй из таких треугольников имеет тип $(4,5, \geqslant 17)$ или $(5,5, \geqslant 16)$ ввиду отсутствия $(4,5, \leqslant 16)$ - и $(5,5, \leqslant 15)$-граней. Если реб- 
ра пятой грани при $v$ суть $u v, v w$, то степени $u v w$ должны быть $(4,5,4),(4,5,5),(5,5,5)$ или $(\geqslant 16,5, \geqslant 16)$, и в последнем случае эта пятая грань является треугольником по лемме $1 \mathrm{a})$. В этих четырех случаях согласно правилу $\mathrm{R} 2 v$ раздает в сумме не более $4 \cdot 1+0=4,3 \cdot 1+\frac{3}{4}+\frac{1}{4}=4,2 \cdot 1+2 \cdot \frac{3}{4}+\frac{1}{2}=4$ и $4 \cdot 1+0=4$. Этим завершается рассмотрение случая 1 ).

2) $x$ есть грань; $x=f \in F$.

2а) $r(f) \geqslant 6$. Теперь $M(f)=r(f)-6 \geqslant 0$ и $f$ может терять заряд только по правилу R5. Инщидентная 6-вершина $v=y$ вносит 1 в $M^{*}(f)$ согласно правилу R3, а два инщидентных ей ребра могут вьносить не более $2 \cdot \frac{1}{2}$ согласно правилу R5. Инцидентная $\geqslant 7$-вершина вносит в $M^{*}(f)$ не менее $\frac{1}{2}$ согласно правилу R3, а два инцидентные ей ребра могут выносить не более $2 \cdot \frac{1}{4}$ по правилу R5. Отсюда $M^{*}(f) \geqslant 0$.

2б) $r(f)=5$. Теперь $M(f)=-1$. Для любой вершины $v$ в границе $f$ определим $n(v)$ как чистьй вклад $v$ в $M^{*}(f)$, т.е. заряд, получаемьй $f$ непосредственно от $v$, минус заряд, потерянный $f$ через ребра, соединяющие $v$ с вершинами степени 3 . Рассуждения из подслучая $2 \mathrm{a}$ ) показьвают, что $n(v) \geqslant 0$ для любой $v$. Если $d(v) \geqslant 10$ для некоторой $v$, то $n(v) \geqslant 1$ по правилам $\mathrm{R} 3$ и $\mathrm{R} 5$, и поэтому $M^{*}(f) \geqslant 0$. Следовательно, мы можем предположить, что $d(v) \leqslant 9$ для любой $v$.

ТАБЛИЦА 2

\begin{tabular}{|c|c|c|c|}
\hline$d(u)$ & $d(v)$ & $d(w)$ & $n(v)$ \\
\hline 3 & 6 & 3 & $\geqslant 1-2 \cdot \frac{1}{2}=0$ \\
3 & 7 & 3 & $\geqslant \frac{1}{2}-2 \cdot \frac{1}{4}=0$ \\
3 & 8 & 3 & $\geqslant 1-2 \cdot \frac{1}{4}=\frac{1}{2}$ \\
3 & 9 & 3 & $\geqslant 1-2 \cdot \frac{1}{6}=\frac{2}{3}$ \\
3 & 10 & 3 & $\geqslant \frac{7}{5}-2 \cdot \frac{1}{10}>1$ \\
3 & $\geqslant 11$ & $\geqslant 3$ & $\geqslant 1-0=1$ \\
\hline
\end{tabular}

\begin{tabular}{|c|c|c|c|}
\hline$d(u)$ & $d(v)$ & $d(w)$ & $n(v)$ \\
\hline 3 & 6 & $\geqslant 4$ & $\geqslant 1-\frac{1}{2}=\frac{1}{2}$ \\
3 & 7 & 4,5 & $\geqslant \frac{1}{2}-\frac{1}{4}=\frac{1}{4}$ \\
3 & 7 & $\geqslant 6$ & $\geqslant \frac{3}{4}-\frac{1}{4}=\frac{1}{2}$ \\
3 & 8 & $\geqslant 4$ & $\geqslant 1-\frac{1}{4}=\frac{3}{4}$ \\
3 & 9 & $\geqslant 4$ & $\geqslant 1-\frac{1}{6}=\frac{5}{6}$ \\
3 & 10 & $\geqslant 4$ & $\geqslant \frac{7}{5}-\frac{1}{10}>1$ \\
\hline
\end{tabular}

Пусть $u v w$ - три последовательные вершины в $f$. Если они имеют степени $(4,5,4)$ или $(5,5,4)$, как в правиле R2 a) или R2б), то вместе дают $f$ не меньше $\frac{1}{2}+0+\frac{1}{2}$ или $\frac{1}{4}+\frac{1}{4}+\frac{1}{2}$, и поэтому $M^{*}(f) \geqslant 0$. Итак, мы можем предположить, что правила R2 a) и $\mathrm{R} 2$ б) не возникают. Поэтому $n(v) \geqslant \frac{1}{2}$, если $d(v)=4$ или 5 . Если $d(v) \geqslant 6$, то снова очевидно, что $n(v) \geqslant \frac{1}{2}$, если ни один из соседей $v$ в $f$ не имеет степени 3, или если $\Delta=11$ (тогда правило R5 не возникает). Если $\Delta \geqslant 12$ и у $v$ есть сосед со степенью 3 , то нижняя оценка для $n(v)$ приводится в табл. 2. Следовательно, если ни одна из вершин $u, v, w$ не имеет степени 3 , то $n(u)+n(v)+n(w) \geqslant \frac{1}{4}+\frac{1}{2}+\frac{1}{4}=1$ и $M^{*}(f) \geqslant 0$. Поэтому мы можем предположить, что в $f$ имеются две несмежные по циклу 3 -вершины, если $\Delta \geqslant 12$, и не меньше четырех 3 -вершин, если $\Delta=11$.

С другой стороны, ввиду отсутствия $(3,3,3,3, \leqslant 9)$-граней $f$ имеет не более трех 3 -соседей, поэтому $\Delta \geqslant 12$. Если в точности три, то ввиду отсутствия $(3,3,3, \leqslant 7, \leqslant 9)$-граней она имеет двух $\geqslant 8$-соседей, для каждого из которых $n(v) \geqslant \frac{1}{2}$ из табл. 2 , так что $M^{*}(f) \geqslant 0$. Итак, мы можем предположить, что $f$ имеет ровно двух несмежных друг с другом 3-соседей. Пусть $f$ имеет вид $u v w x y$, где $d(u)=d(x)=3$. Из табл. 2 имеем $n(v) \geqslant \frac{1}{2}$ и $n(w) \geqslant \frac{1}{2}$ за исключением случая $d(v)=7$, а $d(w) \leqslant 5$ (или наоборот). Но тогда $d(y) \geqslant 8$ из-за отсутствия $(3,3, \leqslant 5, \leqslant 7,7)$-граней, так что $n(y) \geqslant \frac{1}{2}$ и $n(w) \geqslant \frac{1}{2}$. Итак, $M^{*}(f) \geqslant 0$ во всех случаях.

2в) $r(f)=4$. Теперь $M(f)=-2$. Определим $n(v)$ так же, как в подслучае 2в). 
Если в $f$ имеются три последовательные вершины со степенями $(4,5,4)$ или $(5,5,4)$, то, как в подслучае 2в), они вместе приносят $f$ по меньшей мере 1 ; более того, ввиду отсутствия $(4, \leqslant 5,5, \leqslant 10)$-граней оставшаяся вершина имеет степень не меньше 11 и поэтому также вносит вклад 1 по правилу $\mathrm{R} 3$, так что $M^{*}(f) \geqslant 0$. Итак, мы можем предположить, что правила $\mathrm{R} 2 \mathrm{a})$ и $\mathrm{R} 2 б)$ не возникают и $n(v) \geqslant \frac{1}{2}$ для любой вершины $v$ на $f$, имеющей $d(v)=5$. Следовательно, если на $f$ нет 3 -вершин, то $n(v) \geqslant \frac{1}{2}$ для любой вершины $v$ на $f$, откуда $M^{*}(f) \geqslant 0$.

Если $\Delta=11$, так что правило R5 не возникает, то

$$
n(v) \geqslant \begin{cases}1, & \text { если } d(v) \geqslant 8, \\ \frac{1}{2}, & \text { если } 4 \leqslant d(v) \leqslant 7 .\end{cases}
$$

Поскольку $(3,3, \leqslant 7, \leqslant 11)$ - и $(3, \leqslant 7, \leqslant 7, \leqslant 7)$-грани отсутствуют, вершины из $f$ дадут $f$ не менее $1+1, \frac{1}{2}+\frac{1}{2}+1$ или $\frac{1}{2}+\frac{1}{2}+\frac{1}{2}+\frac{1}{2}$. В каждом случае $M^{*}(f) \geqslant 0$. Так что можно предположить, что $\Delta \geqslant 12$.

Поскольку $(3,3,3, \leqslant \Delta)$-грани отсутствуют, на $f$ не более двух 3 -вершин. Предположим сначала, что их ровно две, скажем $w, x$, и что другие две вершины $u, v$ имеют $d(u) \leqslant d(v)$. Тогда $d(u)+d(v) \geqslant 20$ (так как $\Delta \geqslant 12)$. Поэтому если $d(u)=4$ или 5 , то $d(v) \geqslant 15$ и $v$ вносит $\frac{3}{2}$ по правилу $\left.\mathrm{R} 3 \mathrm{a}\right)$, тогда как $u$ вносит $\geqslant \frac{1}{2}$ по правилам $\mathrm{R} 1$ и $\mathrm{R} 2$. Если $d(u) \geqslant 10$, то каждая из $u$ и $v$ вносит $\geqslant 1$ согласно табл. 2 . Поэтому можно считать, что $6 \leqslant d(u) \leqslant 9$ и $d(v) \geqslant 11$. Если $u, v$ последовательны, то $n(v) \geqslant \frac{3}{2}$ по правилу R3 a) и $n(u) \geqslant \frac{1}{2}$ по правилам R3 и R5. Допустим, что это не так, т.е. $f=x u v w$. Тогда $u$ не передает ничего через $u x$ и $u w$ по правилу $\mathrm{R} 5$, так что $n(u) \geqslant 1$ при $d(u) \neq 7$ по правилу R3. Так как $n(v) \geqslant 1$, остается рассмотреть случай $d(u)=7$, в котором имеем $n(u) \geqslant \frac{1}{2}$ по правилу $\mathrm{R} 3$, а $n(v)=\frac{3}{2}$ по правилу $\left.\mathrm{R} 3 \mathrm{a}\right)$, поскольку $d(v) \geqslant 12$ ввиду отсутствия $(3,7,3, \leqslant 11)$-граней. Во всех вариантах имеем $M^{*}(f) \geqslant 0$. Итак, можно считать, что $f$ инцидентна ровно одной 3 -вершине.

Допустим $f=x u v w$, где $d(x)=3$, и $d(u) \leqslant d(w)$. Тогда

$$
d(u)+d(v)+d(w) \geqslant 23
$$

поскольку $\Delta \geqslant 12$. Будем писать $n(u v w)$ вместо $n(u)+n(v)+n(w)$. Если $d(v) \geqslant 6$, то $n(u) \geqslant \frac{1}{2}$ и $n(w) \geqslant \frac{1}{2}$ по правилам $\mathrm{R} 1, \mathrm{R} 2$ и табл. 2 , а $n(v) \geqslant 1$ по правилу $\mathrm{R} 3$, если только степени $u, v, w$ не $(\leqslant 5,7, \leqslant 13)$. Но в последнем случае $d(w) \geqslant 11$ согласно (5) и $n(u v w) \geqslant \frac{1}{2}+\frac{3}{4}+1>2$ по правилам $\mathrm{R} 2, \mathrm{R} 3$ в) и табл. 2 , так что по-прежнему $M^{*}(f) \geqslant 0$. Итак, можно считать, что $4 \leqslant d(v) \leqslant 5$. Ясно, что $d(u) \leqslant 9$ по лемме 1 a). Если $d(w) \leqslant 9$, то $d(u)=d(w)=9$ и $d(v)=5$ согласно $(5)$, а $n(u v w) \geqslant \frac{5}{6}+\frac{1}{2}+\frac{5}{6}>2$ по табл. 2 и правилу R2. Допустим, что $d(w) \geqslant 10$. Тогда $n(u v w) \geqslant \frac{1}{2}+\frac{1}{2}+1$ по правилам $\mathrm{R} 1, \mathrm{R} 2$ и табл. 2 , кроме случая $d(u)=7$. Но $d(u)=7$ влечет $d(w) \geqslant 11$ согласно $(5)$, так что ничего не передается через ребро $x u$ по правилу R 5, и поэтому мы снова имеем $n(u v w) \geqslant \frac{1}{2}+\frac{1}{2}+1$ по правилам R1, R2 и табл. 2 . Во всех вариантах $M^{*}(f) \geqslant 0$.

ТАБЛИЦА 3

\begin{tabular}{|c|c|c|c|c|}
\hline$d(x)$ & $d(y)$ & $d(z)$ & $M^{*}(f)-M(f)$ & по правилу \\
\hline 5 & 10 & $\geqslant 10$ & $\frac{1}{2}+\frac{7}{5}+\frac{7}{5}$ & $\mathrm{R} 2, \mathrm{R} 4, \mathrm{R} 4$ \\
5 & $6-9$ & $\geqslant 11$ & $\frac{1}{2}+1+\frac{3}{2}$ & $\mathrm{R} 2, \mathrm{R} 4, \mathrm{R} 4$ \\
5 & 5 & $\geqslant 11$ & $\frac{3}{4}+\frac{3}{4}+\frac{3}{2}$ & $\mathrm{R} 2$ в $), \mathrm{R} 2$ в $), \mathrm{R} 4$ \\
4 & $6-10$ & $\geqslant 11$ & $\frac{1}{2}+1+\frac{3}{2}$ & $\mathrm{R} 1, \mathrm{R} 4, \mathrm{R} 4$ \\
4 & 5 & $\geqslant 11$ & $\frac{1}{2}+1+\frac{3}{2}$ & $\mathrm{R} 1, \mathrm{R} 2$ г), $\mathrm{R} 4$ \\
\hline
\end{tabular}


2г) $r(f)=3$. Теперь $M(f)=-3$. Пусть $f=x y z$, где $d(x) \leqslant d(y) \leqslant d(z)$. Если $d(x) \geqslant 6$, то каждая вершина дает 1 на $f$ по правилу $\mathrm{R} 4$, а если $d(x) \leqslant 5$ и $d(y) \geqslant 11$, то и $y$ и $z$ дают не менее $\frac{3}{2}$ по правилу R4. Итак, можно считать, что $d(x) \leqslant 5$ и $d(y) \leqslant 10$. Ввиду отсутствия $(5, \leqslant 9, \leqslant 10)-,(4, \leqslant 10, \leqslant 10)$ - и $(4,4, \leqslant \Delta)$-граней варианты с $d(x)=4$ или 5 даны в табл. 3 ; в каждом из них $M^{*}(f)-M(f) \geqslant 3$.

ТАБЛИЦА 4

\begin{tabular}{|c|c|c|}
\hline$d(y)$ & $d\left(z^{*}\right) \geqslant 11$ & $d\left(z^{*}\right) \leqslant 10$ \\
\hline 6 & $1+2$ & $1+\frac{3}{2}+\frac{1}{2}$ \\
7 или 8 & $\frac{5}{4}+\frac{7}{4}$ & $\frac{5}{4}+\frac{3}{2}+\frac{1}{4}$ \\
9 & $\frac{4}{3}+\frac{5}{3}$ & $\frac{4}{3}+\frac{3}{2}+\frac{1}{6}$ \\
10 & $\frac{7}{5}+\frac{8}{5}$ & $\frac{7}{5}+\frac{3}{2}+\frac{1}{10}$ \\
\hline
\end{tabular}

Остается рассмотреть случай $d(x)=3$. Вспомним, что $d(y) \leqslant 10$. Поскольку $(3, \leqslant 5$, $\leqslant \Delta)$ - и $(3, \leqslant 10, \leqslant 12)$-грани отсутствуют, должно быть $6 \leqslant d(y) \leqslant 10$ и $\Delta \geqslant d(z) \geqslant 13$, так что $\Delta \geqslant 12$. Если грань $f^{*}=z^{*} x y \ldots$, смежная с $f$ по ребру $x y$, имеет $d\left(z^{*}\right) \geqslant 11$, то грань $z^{*} x z$ по лемме 1 является треугольником и по правилу R4 $y$ и $z$ дают $f$ суммы, указанные во втором столбце табл. 4 . Если $d\left(z^{*}\right) \leqslant 10$, то $f^{*}$ - нетреугольник, посколькy $(3, \leqslant 10, \leqslant 10)$-грани отсутствуют, и $y, z$ по правилу R5 дают $f$ суммы из третьего столбца табл. 4. Каждая из этих сумм равна 3 и, тем самым, теорема 2 доказана.

\section{СПИСОК ЦИТИРОВАННОЙ ЛИТЕРАТУРЫ}

[1] Steinitz E. Polyheder und Raumeinteilungen // Enzykl. Math. Wiss. 3 (Geometrie). Part 3AB. 1922. V. 12. P. 1-139.

[2] Lebesgue H. Quelques conséquences simples de la formule d'Euler // J. Math. Pures Appl. 1940. V. 9. P. 27-43.

[3] Бородин О.В.Строение и раскраска плоских графов. Дисс. . . . д. ф.-м.н. Новосибирск: ИМ СО РАН, 1994.

[4] Бородин О.В. Строение окрестности ребра в плоском графе и совместная раскраска вершшн, ребер и граней // Матем. заметки. 1993. Т. 53. № 5. С. 35-47.

[5] Иванов А.Б. Полуправильные многогранники // Математическая энциклопедия. Т. 4. М.: Советская энциклопедия, 1984. С. 463.

[6] Kotzig A. Extremal polyhedral graphs // Proc. 2nd Intern. Conference on Combinatorial Math. New York, 1978. P. 569-570.

[7] Бородин О.В.Минимальный вес грани в плоских триангуляциях без 4-вершин // Матем. заметки. 1992. Т. 51. №1. С. 16-19.

[8] Borodin O. V. Triangulated 3-polytopes with restricted minimal weight of faces // Discrete Math. (to appear).

[9] Borodin O. V. Cyclic degree and cyclic coloring of 3-polytopes // J. Graph Theory. 1996. V. 23. № 3. P. 225-231.

[10] Hor n̆ák M., Jendrol' S. Unavoidable sets of face types for planar maps // Discuss. Math. (to appear).

[11] Borodin O. V., Woodall D. R. Cyclic degrees of 3-polytopes // SIAM J. Discrete Math. (to appear).

[12] Бородин О. В. Совместное обобщение теорем Лебега и Коцига о комбинаторике плоских карт // Дискретная матем. 1991. Т. 3. № 4. С. 24-28. 